\title{
ARCADE: Description of the project and setup of the Lidar/AMT system
}

\author{
L. Valore ${ }^{1, \text { a }}$, M. Buscemi ${ }^{1}$, C. Cassardo ${ }^{2}$, M. Cilmo ${ }^{1}$, M. Coco $^{2}$, S. Ferrarese ${ }^{2}$, F. Guarino ${ }^{1}$, \\ M. Iarlori ${ }^{3}$, H.J. Mathes ${ }^{4}$, V. Rizi ${ }^{3}$, A.S. Tonachini ${ }^{2}$, L. Wiencke ${ }^{5}$, and M. Will, b \\ ${ }^{1}$ Universita' degli Studi di Napoli "Federico II" \\ ${ }^{2}$ Universita' di Torino \\ ${ }^{3}$ CETEMPS/DSFC Universita' degli Studi dell'Aquila \\ ${ }^{4}$ Karlsruhe Institute of Technology \\ ${ }^{5}$ Colorado School of Mines, Golden, Colorado
}

\begin{abstract}
The ARCADE (Atmospheric Research for Climate and Astroparticle DEtection) project is a 3 years project funded by MIUR, that aims to study the aerosol attenuation of UV light in atmosphere using multiple instruments and techniques, as those commonly used in the cosmic rays community: elastic Lidar, Raman Lidar, side-scattering measurements using a distant laser source. All measurements will be acquired on the same air mass at the same time, in a semi-desertic site near Lamar, Colorado (U.S.). For each instrument, multiple analysis techniques will be tested: the target is a better comprehension of the systematics and limits of applicability of each method. The system is composed by a Lidar (elastic+Raman), fully designed and built within this project, and by the Atmospheric Monitoring Telescope (AMT), a telescope for the detection of UV light owned by the Colorado School of Mines. The setup of the two instruments is described in detail here. The project is presently in its third year: the Lidar system has been tested at the University of L'Aquila in February 2014 before shipment to the U.S., and the AMT has been recently reinstalled and tested in Lamar (May 2014). In June/July 2014 the ARCADE group will work out the final setup of the Lidar+AMT system in Lamar and will begin data acquisition.
\end{abstract}

\section{Introduction}

The ultra high energy cosmic rays primary particles entering the atmosphere, while interacting with air molecules, produce cascades of secondary particles named Extensive Air Showers (EAS). During the development of the shower, a certain amount of fluorescence light (UV light in the range $300-420 \mathrm{~nm}$ ) is emitted isotropically. The fluorescence light can be detected at ground level and leads information about the primary particle that produced the EAS. In this scenario, the atmosphere has a crucial role: it is responsible for both the production and the attenuation of the fluorescence light during its travel towards the detector. Those experiments studying ultra high energy cosmic rays by detecting the fluorescence light produced during the EAS development need to perform a continuous and reliable monitoring of the properties of the atmosphere during data acquisition: in particular, atmospheric aerosols need to be constantly monitored since they are the most variable component in this scenario, both in distribution and time, on a time scale as little as one hour. The ARCADE collaboration is composed of a small group of physicists that have a long time experience in the atmospheric monitoring for high energy cosmic rays experiments: the experience gained in the measurement of the aerosol attenuation profiles of the UV light in atmosphere for cosmic rays experiments made clear the need for a better comprehension of the systematics, limits of applicability and compatibility of the results obtained

\footnotetext{
${ }^{a}$ e-mail: laura.valore@na.infn.it

${ }^{\mathrm{b}}$ Now at Instituto de Astrofísica Canarias, La Laguna, Tenerife, Spain.
}

with the typical instruments and techniques used in this field, and ARCADE aims to address these points. The instruments typically used in cosmic rays experiments to measure the aerosol attenuation profiles of the fluorescence light are Lidars (elastic and Raman) [1] and Distant Laser Facilities [2]. The ARCADE system is composed by a Lidar, fully designed and built within this project, and by the Atmospheric Monitoring Telescope (AMT), a telescope to detect the UV light emitted by the Lidar laser source at a distance of $39.8 \mathrm{~km}$. The Lidar system construction has been completed and it has been tested at CETEMPS/DSFC of University of L'Aquila in February 2014 in parallel with a well tested Lidar system which is one of the stations of the EARLINET network (European Aerosol Research Lidar Network) [3]. The tests evidenced the need for some refinement that has been done before the shipment to Lamar, Colorado, in May 2014. In June 2014, the Lidar system will be installed on site. During May 2014 the AMT has been tested at the Colorado School of Mines in Golden, Colorado and has been reinstalled on site in Lamar. In the following section, the setup of the ARCADE system, tests and installation will be described.

\section{Target of the project and location}

The ARCADE project will collect data for the first time simultaneously with multiple instruments and using different analysis techniques as those commonly used in the cosmic rays community. The target is to measure the aerosol attenuation profiles and systematically compare results for a better knowledge of the systematics and limits of applicability of each technique. The project also aims to develop models that describe the stratification of 


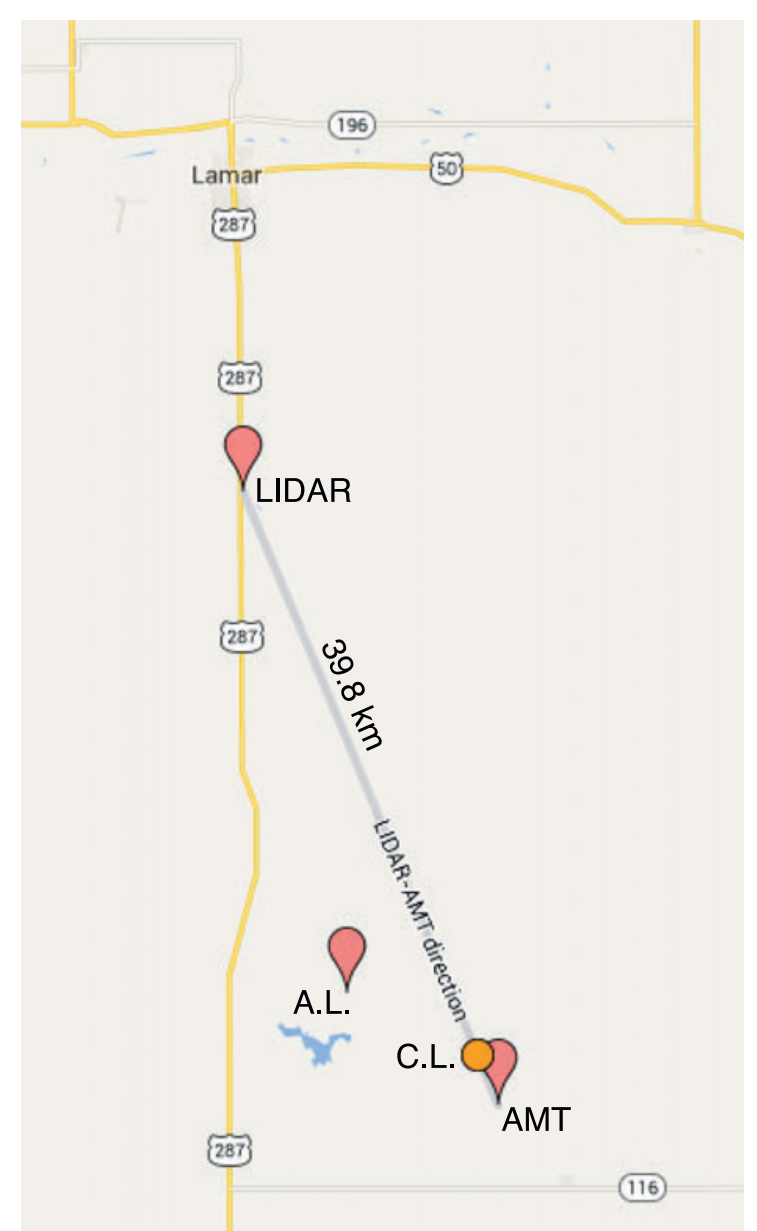

Figure 1. ARCADE location (Google Maps). The distance between the two telescopes (Lidar and AMT) is $39.8 \mathrm{~km}$, long enough that no direct line of sight is between the two sites because of the curvature of Earth. A location on top of a hill (A.L.) has been used to align the AMT field of view to the Lidar location. Another location, marked with an orange circle, has been used to calibrate the AMT with a low energy laser in the field (C.L.).

aerosols and their dependence on temperature, wind, rain and humidity, in collaboration with climatology experts that will apply predictive models to these measurements. A study of the cloud cover and cloud optical depth is also going to be performed.

The location chosen is an arid environment with low pollution, the typical characteristics required for a cosmic rays detectors site. The site chosen is SouthEastern Colorado, near the town of Lamar, $350 \mathrm{~km}$ south of Denver, a flat plateau at about $1200 \mathrm{~m}$ above sea level. Infrastructures for atmospheric research were already present on site: the AMT, owned by the Colorado School of Mines, was already used in 2010-11 and it has been upgraded and reinstalled on site for this project. In Fig. 1 a map of the site with the positions of the Lidar and the AMT is shown.

\section{The Lidar}

The ARCADE Lidar has been designed and realized completely in Italy within this project, mainly at the INFN

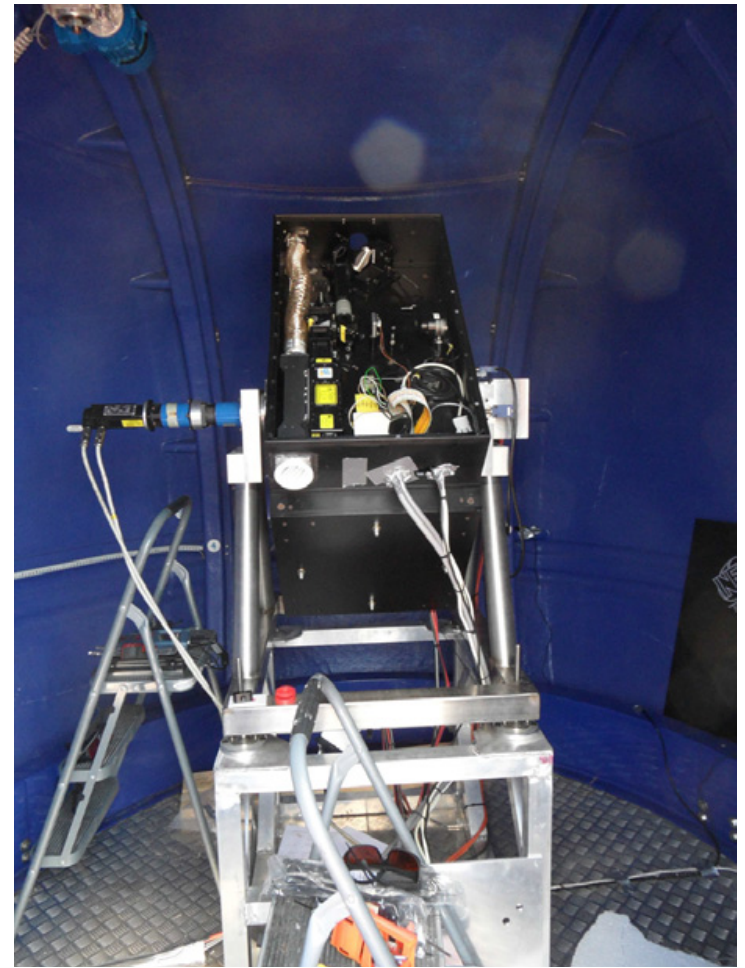

Figure 2. The ARCADE Lidar: the laser bench and the steering mount.

Mechanical Workshop of Torino with support from the INFN Mechanical Workshop of Napoli. It is an extremely compact system of $1.5 \mathrm{~m} \times 0.8 \mathrm{~m}$ that hosts the laser bench, the primary mirror and the receiver box. The system is fixed on a $0^{\circ}$ to $90^{\circ}$ zenithal steerable mount and is housed inside an astronomical dome having a diameter of $3 \mathrm{~m}$, positioned on the roof of a 20 feet shipping container. A picture of the Lidar is shown in Fig. 2. The system has been designed to be completely controlled from remote and adopts a certain number of security tools for remote operation.

The laser source is a Quantel Centurion Nd:YAG diode-pumped solid state laser firing at $355 \mathrm{~nm}$ after passing through a second and third harmonic generation modules. Not negligible residuals at 1064 and $532 \mathrm{~nm}$ have been filtered out using 5 dichroic mirrors (one Quantel block containing 4 dichroics plus one standalone dichroic mirror placed right at the exit of the laser box) which guarantee an ultra pure beam. The laser is capable of firing at variable frequencies from $4 \mathrm{~Hz}$ to $100 \mathrm{~Hz}$ : this characteristic is mandatory since the AMT can only acquire signals up to $4 \mathrm{~Hz}$, while higher repetition rates are preferrable to collect more data in a short time with the Raman Lidar.

At the laser exit, the beam size is $\sim 2 \mathrm{~mm}$ and the beam divergence is $3 \mathrm{mrad}$. A beam splitter is used to send $95 \%$ of the beam into a $10 \mathrm{X}$ beam expander that reduces the laser beam divergence to $0.3 \mathrm{mrad}$, and $5 \%$ to a LaserProbe Inc. Rjp-445 pyroelectric energy probe to measure the energy fired at each single shot and record it using a Rm6600 radiometer.

Before exiting the laser box, the beam is depolarized. A two-axis motorized mirror mount, holding a $2^{\prime \prime}$ flat 


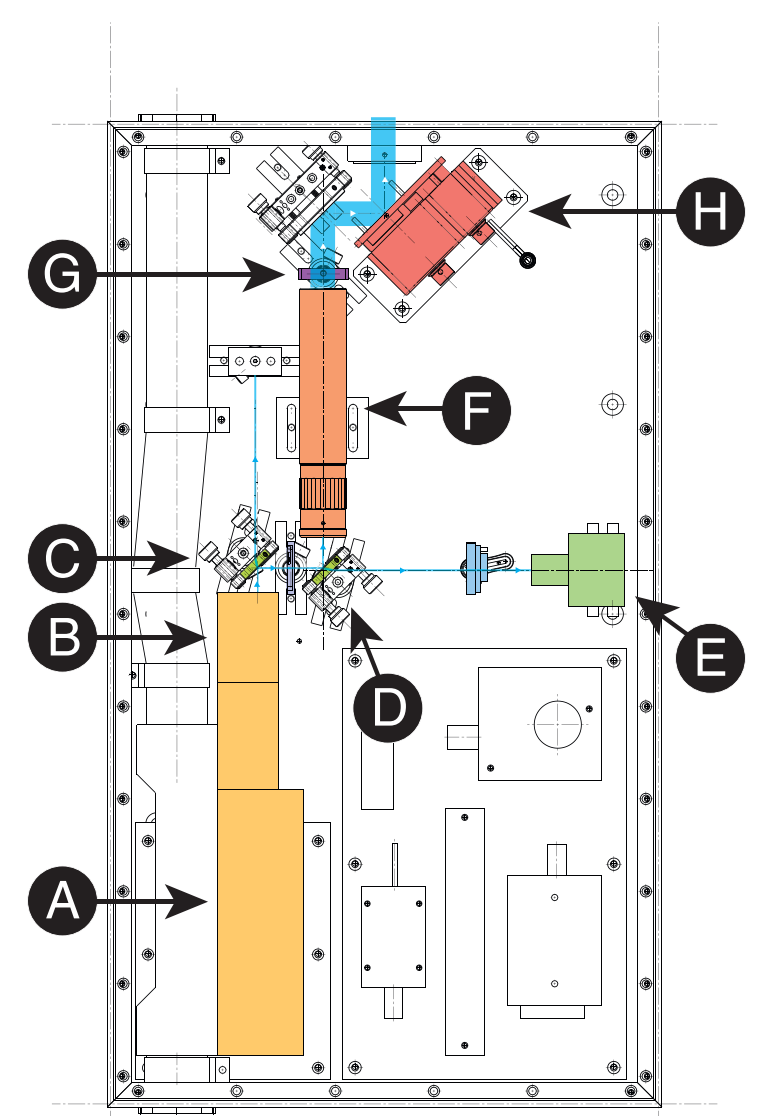

Figure 3. Laser bench scheme. The laser (A) emits $1.6 \mathrm{~mm}$ light beam with a full divergence of $\sim 3 \mathrm{mrad}$. Light is purified by five dichroic mirrors (B,C). A beam splitter (D) sends $5 \%$ of the light to a laser probe $(\mathrm{E})$. The main beam passes though a $10 \mathrm{X}$ beam expander $(\mathrm{F})$, thus reducing the divergence to $\sim 0.3 \mathrm{mrad}$. The light is then depolarized $(\mathrm{G})$. The beam alignment can be finely controlled with a motorized mirror mount $(\mathrm{H})$. The beam finally exits the laser box passing through a quartz window.

mirror, is controlled by computer, and allows fine adjustments of the output beam direction. A sketch of the optical bench is shown in Fig. 3.

The backscattered light is collected by a $25 \mathrm{~cm}$ primary parabolic mirror. It is then deflected by a flat mirror onto a lens that defocuses the light beam. The return light is thus launched on a beam splitter which separates the elastic and Raman backscattered photons. The resulting elastic beam passes through a $354.7 \pm 2 \mathrm{~nm}$ narrowband filter, while the $\mathrm{N}_{2}$ Raman line at $387 \mathrm{~nm}$ is selected by a second narrowband filter. The two beams are read by two separate $2^{\prime \prime}$ R1332 photomultiplier tubes by Hamamatsu. Signals are then amplified 20 times and sampled with a 10 bit 2 GS/s CAEN DT5751 digitizer.

The parallax between the laser beam and the receiver is $301 \mathrm{~mm}$. It is expected from ray tracing simulations to be able to have a complete overlap of the laser beam and the mirror field of view at a distance of about $250 \mathrm{~m}$.

The Lidar is completely controlled by remote: a schematization of all the connections between the computers and hardware is shown in Fig. 4.

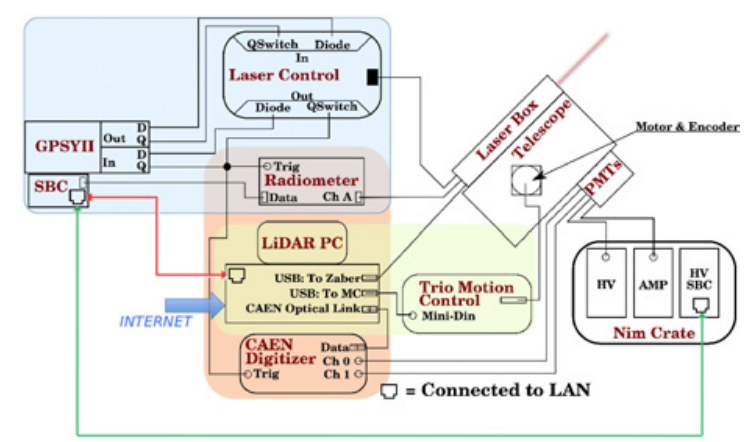

Figure 4. The Lidar scheme.

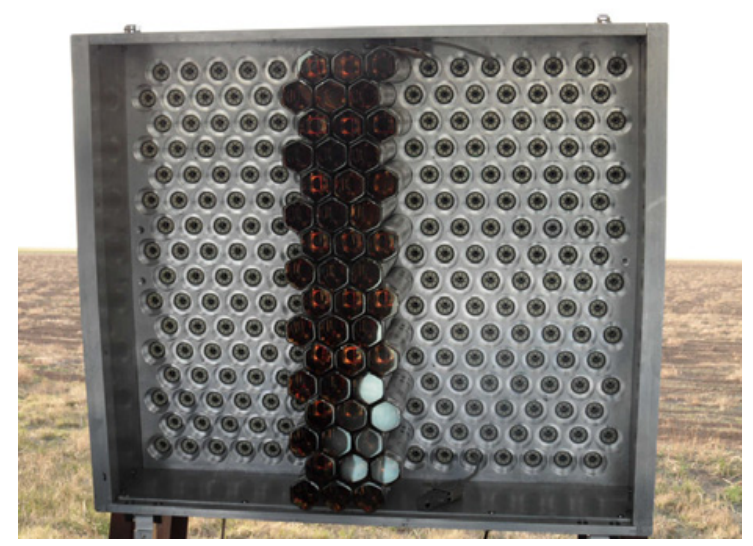

Figure 5. AMT camera equipped with 3 columns of XP3062 photomultipliers.

\section{The Atmospheric Monitoring Telescope (AMT)}

The Atmospheric Monitoring Telescope (AMT) is a telescope for the detection of UV light that was built in 2008 by the Colorado School of Mines within the atmospheric $\mathrm{R} \& \mathrm{D}$ program for the Auger North experiment and it was operated in 2010-2011 [4]. Some parts of the telescope are spares recovered from the HiRes experiment, while other parts, like the housing, have been built for the R\&D program. The telescope is composed by a camera $56 \mathrm{~cm} \times 40 \mathrm{~cm}$ equipped with 3 columns of 16 Photonis XP3062 photomultipliers (PMTs) for the detection of UV light (see Fig. 5), protected by a UV filter. The camera is not fully equipped on purpose: 3 columns of PMTs are sufficient to collect the Lidar tracks.

The light receiver is composed of a 4-segment spherical mirror having a total area of $2 \mathrm{~m}^{2}$ (see Fig. 6). The optics assembly ensures a $1^{\circ} \times 1^{\circ}$ field of view to each PMT. In the middle of the 4 segments mirror an LED facility is installed.

The LED facility was built to emit uniform and stable UV light across the camera, to perform a day by day calibration of the PMTs and electronics response to a fixed amount of light. A photodiode is used to trace the light emitted by the LED.

The AMT telescope is housed in a dedicated waterproof container with authomatic and remotely controlled doors, see Fig. 7. Limit switches are used to safely control the door opening when the AMT is remotely 


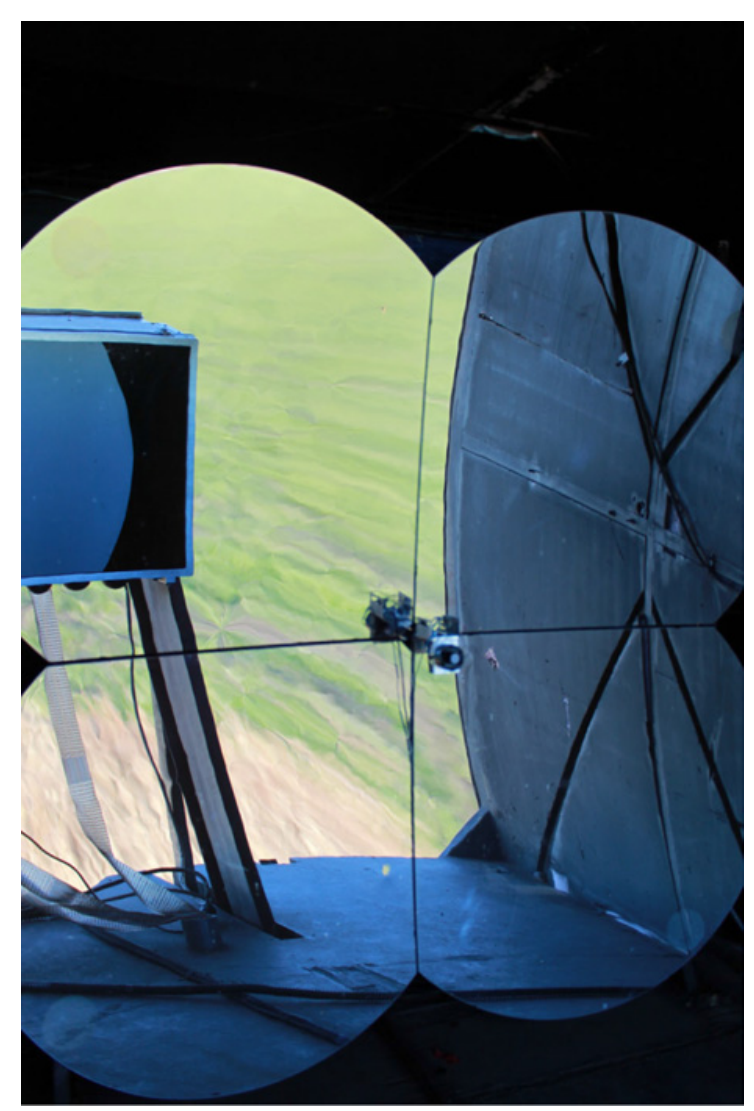

Figure 6. The 4-segment spherical mirror and the LED facility. The camera, equipped with the UV filter, is reflected in the mirror.

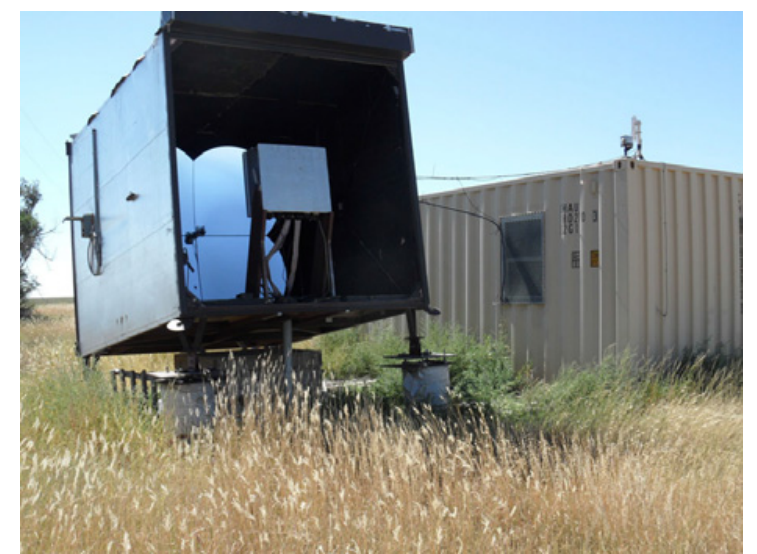

Figure 7. The AMT: on the left, the 4-segment spherical mirror and the camera housed within the waterproof shelter. The office container next to the telescope hosts all the computers.

operated. Concrete pillars and adjustable feets were made to obtain an angle of elevation of the telescope of $8.72^{\circ}$ from the horizontal. The camera can view the laser up to $10.8 \mathrm{~km}$ in height, starting from $1.5 \mathrm{~km}$ from ground level.

Within the ARCADE project, the AMT has been first moved to the laboratory of the Colorado School of Mines in Golden, Colorado, at the end of 2012 to verify and upgrade it after the long period of inactivity. During this period, the Data Acquisition System has been improved and a new LED facility has been installed. Before the reinstallation of the whole system in Lamar, the functionality

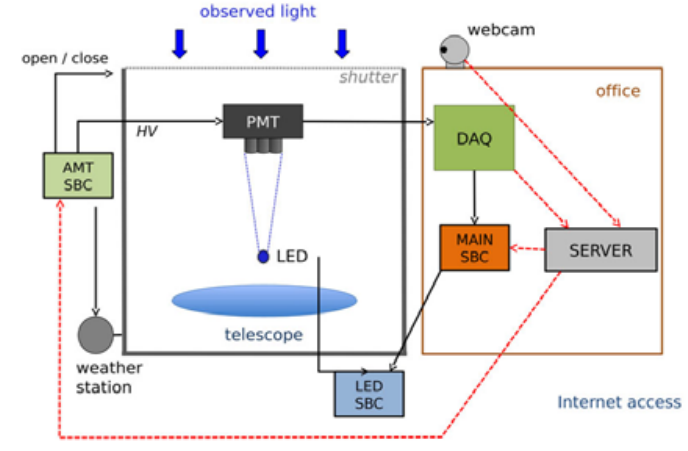

Figure 8. The AMT scheme.

and connections between the 3 Single Board Computers (SBCs), the PC for Data Acquisition (DAQ) and the main server have been tested. A GPS pulse generator based on the GPSY-II board [5] is used to synchronize the data acquisition to the Lidar laser firing time. The Main SBC is used to run the program for the DAQ and is equipped with the GPSY-II board; the AMT SBC controls the telescope container, shutter, PMTs HV modules, camera power and receives informations from the weather stations; the LED SBC drives the LED facility. The full scheme is shown in Fig. 8.

The reinstallation of the AMT in Lamar, near the site of Two Buttes, took place in May 2014. The functionality of the whole camera of PMTs and LED system have been tested on site, as well as the external access to the main server and all the SBCs for remote operation. Two UPS systems are used to prevent the computers to shutdown in case of temporary power failures. Since the AMT is operated remotely, a number of safety checks (power, wind speed, rain, twilight time) are permanently running during data taking to ensure the closure of the telescope in case of failures or unsecure conditions.

\section{The next steps}

During June/July 2014 the Lidar will be installed in Lamar. We plan to test the whole AMT and Lidar system, and take the first data with both instruments at the same time. The system will be operated in moonless nights. The groups involved are developing the tools for the data analyses. We plan to apply the multiangle analysis and Raman analysis to the Lidar data, as described in [1], and the Laser Simulation Analysis for the side-scattering measurements performed with the AMT, as described in [2].

\section{References}

[1] The Lidar System of the Pierre Auger Observatory, S.Y. BenZvi et al., Nucl. Instrum. Meth. A 574: 171-184 (2007)

[2] Techniques for measuring aerosol attenuation using the Central Laser Facility at the Pierre Auger Observatory, by The Pierre Auger Collaboration (L. Valore corresponding author). JINST 8, P04009 (2013) 
[3] http://www.earlinet.org/

[4] Atmospheric Super Test Beam for the Pierre Auger Observatory, L. Wiencke for the Pierre Auger Collaboration and A. Botts, C. Allan, M. Calhoun, B. Carande, M. Coco, J. Claus, L. Emmert, S. Esquibel, Hamilton, T.J. Heid, F. Honecker, M. Iarlori,
S. Morgan, S. Robinson, D. Starbuck, J. Sherman, M. Waken, and O. Wolf, Proc 32nd International Cosmic Ray Conference (August, 2011)

[5] J. Smith, J. Thomas, S. Thomas, and L. Wiencke, Proc. 30th ICRC, Merida, Mexico, 5 997-1000 (2007) 